\title{
Development and Application of TL-1 New High Temperature and High Pressure Plugging Instrument
}

\author{
Yanlin Zou, Chunzhi Luo*, Baijing Wang, Huan Zhang, Chujun Zhang \\ College of Chemistry and Environmental Engineering, Yangtze University, Jingzhou, China \\ Email: *405053099@qq.com
}

How to cite this paper: Zou, Y.L., Luo, C.Z., Wang, B.J., Zhang, H. and Zhang, C.J. (2021) Development and Application of TL-1 New High Temperature and High Pressure Plugging Instrument. Open Journal of Yangtze Gas and Oil, 6, 107-115.

https://doi.org/10.4236/ojogas.2021.63010

Received: June 21, 2021

Accepted: July 13, 2021

Published: July 16, 2021

Copyright $\odot 2021$ by author(s) and Scientific Research Publishing Inc.

This work is licensed under the Creative

Commons Attribution International

License (CC BY 4.0).

http://creativecommons.org/licenses/by/4.0/

\begin{abstract}
In view of the fact that most of the filter media of existing plugging evaluation instruments use steel plates or steel balls, which cannot truly simulate the characteristics of the formation, a new type of TL-1 high temperature and high pressure plugging instrument has been developed. The filter medium of the instrument is the natural stone with different cracks and quartz sand beds with different particle sizes, which can better simulate the adsorption and hang-up effect of the formation on the plugging agent. It is possible to evaluate the plugging effect, pressure-bearing capacity and return pressure of the plugging slurry for different cracks and sand beds of different sizes under different temperatures and pressures. Using new and old instruments to evaluate the plugging effect of the same plugging slurry, it is found that the plugging effect is different from the distribution of the plugging material on the crack surface, the plugging effect and the pressure bearing capacity. The instrument is stable and reliable in operation and simple in operation. It is a new instrument for conducting research on anti-leakage plugging materials and anti-leakage plugging drilling fluid systems.
\end{abstract}

\section{Keywords}

Plugging Device, Filter Medium, Plugging Experiment, Plugging Formula

\section{Introduction}

Lost circulation is one of the more common downhole complex conditions in the oil drilling process, and it has the characteristics of universality, variability, and complexity. Lost circulation not only loses drilling fluid, consumes drilling time, and prolongs the drilling cycle, but also may cause a series of complex downhole accidents such as well collapse, blowout, and sticking, causing signifi- 
cant economic losses [1] [2]. Therefore, the scientific and efficient selection of plugging materials and the preparation of plugging slurry for rapid plugging are crucial to the success of plugging. At present, plugging is generally based on experience to select various materials and methods at the site, and small leakage may be easier to solve; For the unstable leakage of the formation itself, if the leakage cannot be successfully plugged quickly, it will cause the collapse of the well wall and the sticking of the drill due to the long leakage time. It is also common that after successful plugging at the site, the circulation of the drilling fluid increases or the drilling speed is too fast, when the suction effect is generated, the plugging material returns to spit, and the leakage occurs when recirculating drilling. Therefore, it is very important for safe drilling to make a leak-proof and plugging plan for the easy-leakage and easy-to-collapse formations in advance. The ability of plugging materials to penetrate deep into the cracks or pores and retain them is an important factor in the success of plugging. The plugging evaluation instrument is an important instrument for us to screen materials and do a good job of preventing leakage and plugging. Domestic and international plugging evaluation instruments mainly include API static plugging evaluation instrument, DL type plugging instrument, high temperature and high pressure dynamic plugging instrument, high pressure crack test instrument, high temperature and high pressure drilling fluid loss dynamic evaluation instrument, etc. [3], These instruments all use steel plate cracks or steel balls as filter media, and cannot truly simulate the surface properties of cracks and pores in the downhole formation. The experimental research results are of little significance to the field. In this paper, TL-1 new high temperature and high pressure plugging instrument was developed, mainly for the development and application of leakage prevention and plugging technology on the south bank of Tahe. It can not only simulate the formation temperature and pressure but also simulate the surface characteristics of formation cracks and pores. It can also observe the distribution of the plugging material entering the cracks and determine the pressure required for regurgitation. The researched plugging formulas and construction plans have a good guiding significance for on-site leakage prevention and plugging construction.

\section{Instrument Composition and Working Principle}

\subsection{Composition of the Instrument}

TL-1 new plugging instrument consists of gas source, ring pressure pump, mud tank, high pressure relief valve, filtered core medium formed by natural rock or quartz sand with different cracks, pressure gauge, back pressure device, heating control system, temperature Sensors, mud recovery tanks, various pipelines and other parts. The overall structure is shown in Figure 1, and the physical map is shown in Figure 2.

\subsection{Principles and Steps of Instrument Experiment}

At a certain temperature, pressurize a mud tank with a certain volume, and the 

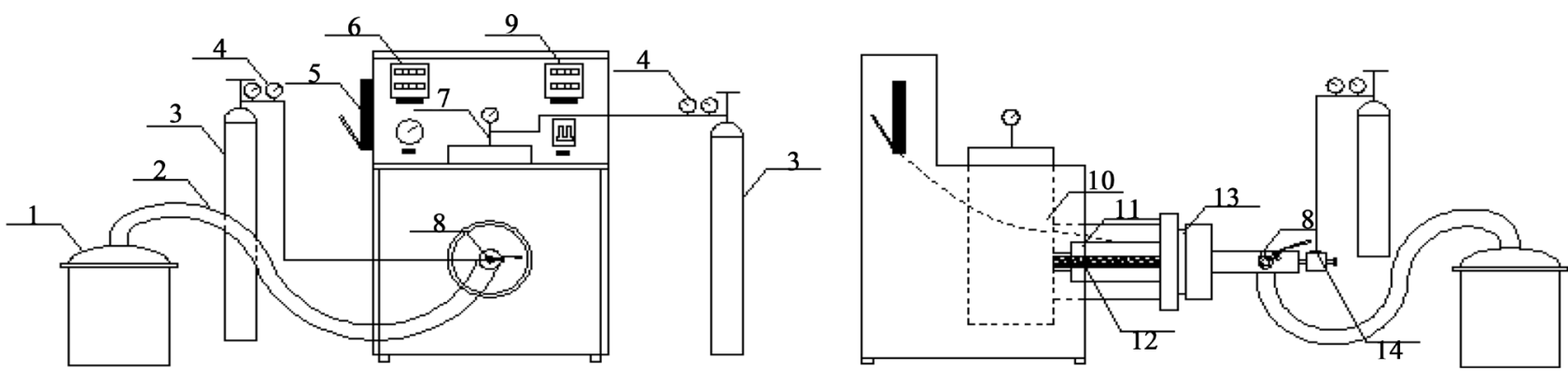

1-Mud recovery tank; 2-Pipeline; 3-Cylinder; 4-Reducing valve; 5-Ring pressure pump; 6-Temperature setting controller; 7-Pressure gauge; 8-Three-way valve; 9-Real-time temperature display; 10-Mud tank; 11-Core holder; 12-Simulated core or quartz sand tube; 13-Core or quartz sand tube fixing device; 14-Return exhaust pressure test inlet.

Figure 1. The overall structure of the TL-1 new plugging instrument.

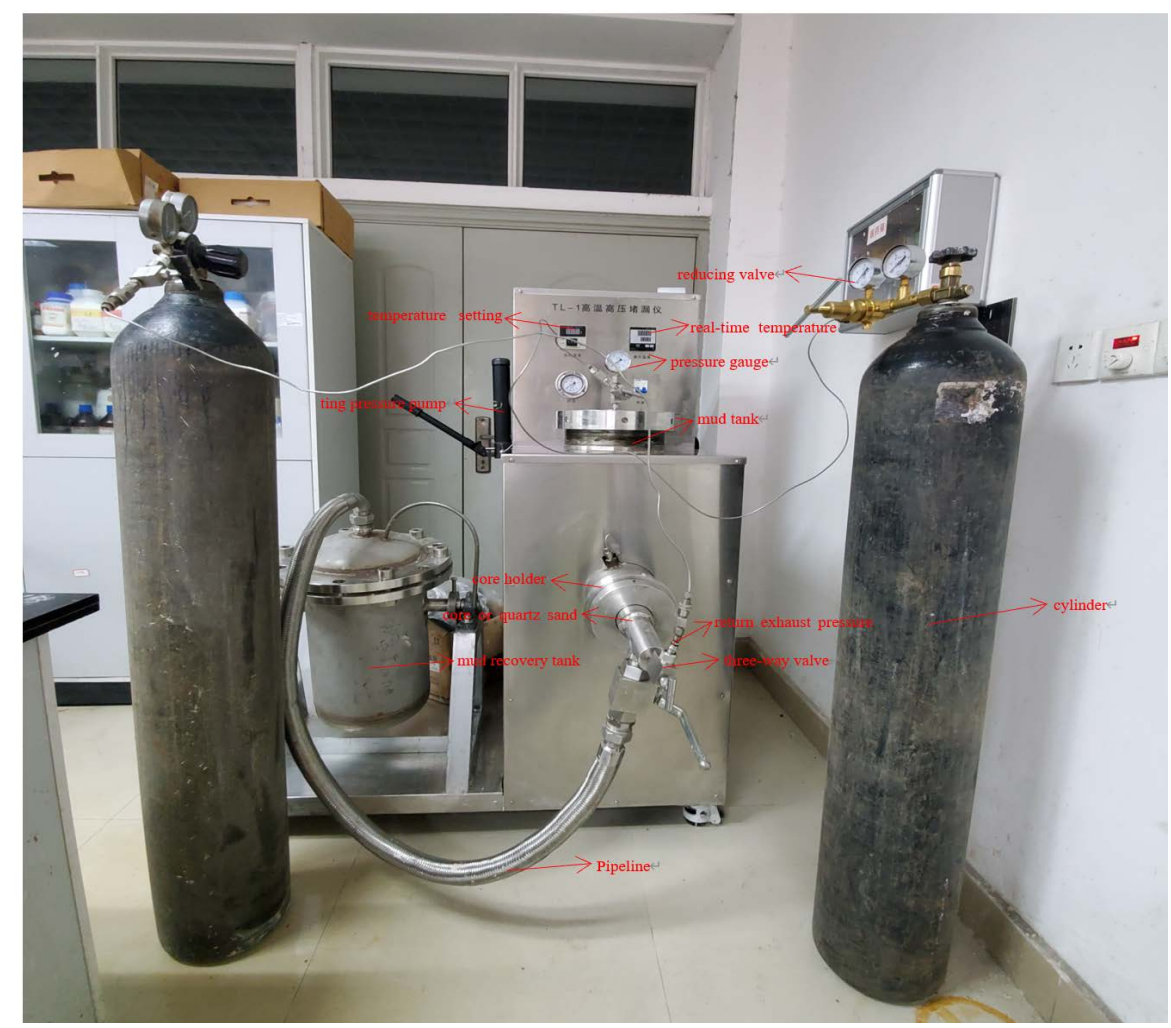

Figure 2. Physical image of TL-1 new plugging instrument.

drilling fluid will seep into the pores and cracks of the core, causing leakage or loss. By observing the pressure change in the mud tank, the amount of mud leakage, and measuring the pressure-bearing capacity of the plugging layer to determine whether the plugging is successful, then the plugging material and the plugging slurry formula are optimized.

The operation steps of TL-1 new high temperature and high pressure plugging instrument are as follows.

1) Select a cracked core of a certain size or a sand-filled pipe with certain particle size, put it into the core holder, and pressurize the core holder through the ring pressure pump, so that the plugging slurry can only be passed from the cracked core or sand-filled pipe under the action of the displacement pressure; 
2) Pour the plugging slurry into the high temperature and high pressure mud tank, tighten the mud tank cover, connect various pipelines, set the experimental temperature, and start heating;

3) After the temperature reaches the set value, open the core holder outlet valve, and then open the nitrogen bottle to pressurize the high temperature and high pressure mud tank, increasing by $0.5 \mathrm{MPa}$ every 20 minutes. At the same time, observe whether the pressure in the mud tank is stable within 20 minutes, whether there is mud leakage from the outlet of the holder;

4) During the experiment, if the pressure in the mud tank has been shown to be 0 , and almost all the mud is observed to leak out, it indicates that the crack or the sand-filled pipe has not been blocked; If the pressure in the mud tank changes simultaneously with the displacement pressure and stabilizes within 10 minutes, no mud leaks out of the core holder outlet, indicating that the plugging is successful under this displacement pressure;

5) if the plugging is successful, close the core holder outlet valve, vent the pressure in the high temperature and high pressure mud tank, then close the mud tank inlet valve, open the core holder outlet end of the flowback inlet valve, and impose flowback pressure on the plugging layer, observe the pressure change on the pressure gauge on the mud tank until the pressure on the mud tank increases, indicating that the plugging layer is broken down by the flowback pressure, and now the flowback pressure is the pressure bearing capacity of the plugging layer;

6) After the experiment is completed, the system pressure is released, the temperature drops to room temperature, the mud tank is opened, the drop height of the plugging slurry level in the tank is measured, and the cumulative leakage is calculated;

7) Take out the core, observe the distribution of the plugging material on the cross section of the core, and take pictures;

8) Pour out the remaining mud, clean the instrument and core, and dry the core for later use.

\section{Instrument Characteristics and Main Indicators}

\subsection{Instrument Characteristics}

1) Different filter media. By comparing the filter media of domestic and foreign plugging evaluation instruments, such as N. Kaageson-Loe [4] and others reported that particles of different particle sizes were filled between two parallel perforated metal plates to simulate crack walls with different permeability; Bingzhong Shi [5] and others used high-precision laser etching technology to precisely etch simulated cracks with various micron-level crack widths in the middle of the tempered glass surface, these steel plates or steel balls are used as filter media, which have poor simulation degree and large experimental errors, which cannot truly reflect the characteristics of the formation. The filter medium of the TL-1 high temperature and high pressure plugging instrument developed 
in this paper is a sand-filled tube formed by natural rocks with different crack sizes and quartz sand with different particle sizes, Figure 3 shows the front of the sand-packed pipe, and Figure 4 shows the end face of the sand-packed pipe, which can simulate formation pores and cracks more realistically. Figure 5 shows a natural core with a length and width of $20 \mathrm{~cm} \times 8 \mathrm{~cm}$, and the crack surface is uneven, which can simulate the surface state of the formation crack more realistically.

2) The surface is hydrophilic. It has a good adsorption and hang-up effect on water-based plugging materials. It can be evaluated and optimized for waterbased drilling fluid plugging materials and plugging slurry formulations; in addition, the core is infiltrated by the wetting reversal agent to transform the surface of the core cracks for the lipophilicity, it is used to evaluate the preferred

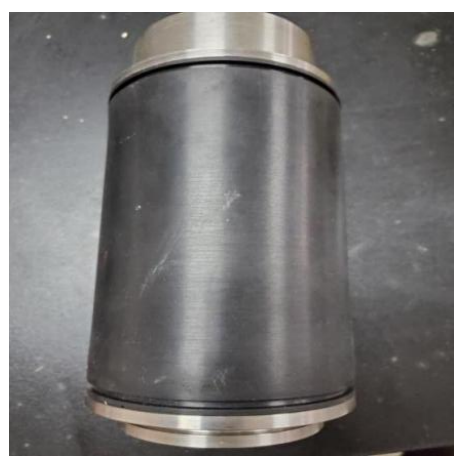

Figure 3. Sand filling pipe.

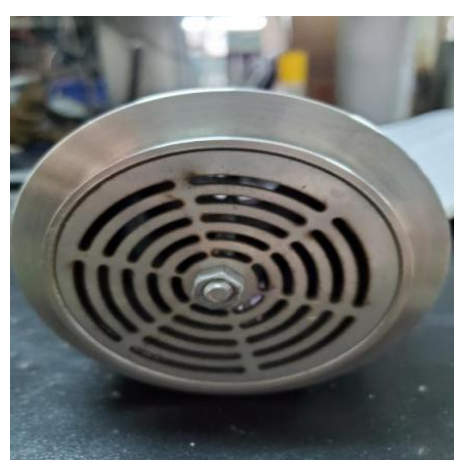

Figure 4. End face of sand-filled pipe.

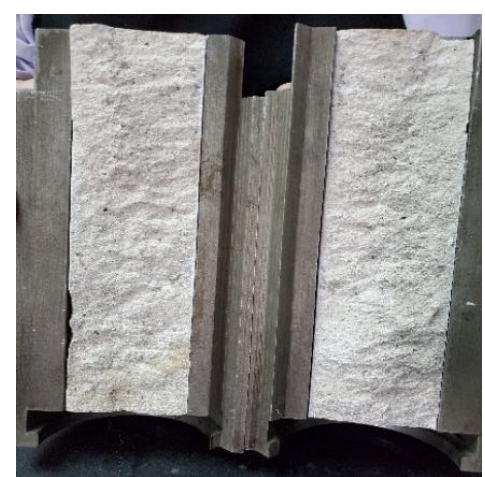

Figure 5. The surface of the core fracture. 
oil-based drilling fluid plugging materials and plugging slurry;

3) After the experiment, the cracks can be opened to observe the distribution of the plugging material on the surface of the core;

4) The size of the fractured core can be adjusted, the opening is $1-15 \mathrm{~mm}$, as shown in Figure 6 is a $2 \mathrm{~mm}$ fractured core;

5) It can measure the experimental parameters such as the maximum pressure at which leakage occurs, the pressure-bearing capacity after successful plugging, and the cumulative leakage;

6) The experiment temperature and pressure can be adjusted according to actual needs.

\subsection{Main Indicators}

1) Temperature: 0 - $200^{\circ} \mathrm{C}$;

2) Working pressure: 0 - $20 \mathrm{MPa}$;

3) Flowback pressure: 0 - $20 \mathrm{MPa}$;

4) The amount of plugging slurry: $5 \mathrm{~L}$;

5) Heating power: $4 \mathrm{KW}$;

6) Crack width: $1 \mathrm{~mm}, 2 \mathrm{~mm}, 3 \mathrm{~mm}, 4 \mathrm{~mm}, 10 \mathrm{~mm}$;

7) Sand size: choose according to needs.

\section{Instrument Evaluation}

\subsection{Evaluation Index}

The main evaluation indicators of TL-1 high temperature and high pressure plugging instrument:

1) The minimum pressure and time of leakage when the plugging slurry leaks;

2) Loss of plugging slurry;

3) The pressure-bearing capacity of the plugging layer.

\subsection{Evaluation of the Experimental Performance of the Instrument}

Refer to the plugging slurry prepared on site for different leakage speeds, three kinds of plugging slurry were prepared in laboratory, TL-1 high temperature and

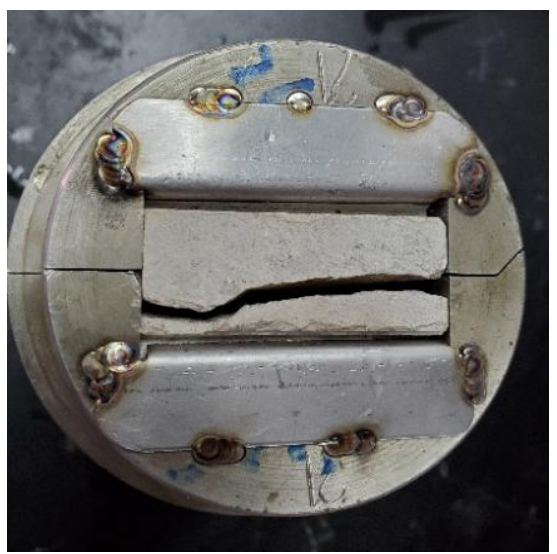

Figure 6. The end face of $2 \mathrm{~mm}$ fractured core. 
high pressure plugging instrument and traditional plugging instrument were used to evaluate the minimum pressure of plugging slurry when plugging cracks with different widths, and how long to stop the leakage under this pressure; The pressure change in the mud tank as the flowback pressure increases after the plugging is successful; After the experiment, observe the distribution state of the plugging material on the crack surface. The experimental results are shown in Table 1, Table 2 and Figure 7, Figure 8. The core crack width and the width between steel plates selected for the experiment are $1 \mathrm{~mm}, 2 \mathrm{~mm}$ and $4 \mathrm{~mm}$.

$1^{\#}$ Plugging slurry: On-site well slurry $+1 \%$ walnut shell (within $1 \mathrm{~mm}$ ) + $0.5 \%$ peanut shell (within $1 \mathrm{~mm}$ ) $+1 \%$ SQD-98 fine $+0.25 \%$ JYW-2.

$2^{\#}$ Plugging slurry: On-site well slurry $+3 \%$ walnut shell (within $2 \mathrm{~mm}$ ) $+0.4 \%$ vermiculite (within $2 \mathrm{~mm}$ ) $+1 \%$ SQD-98 fine + 1\% SQD-98 medium coarse.

$3^{\#}$ Plugging slurry: On-site well slurry $+5 \%$ walnut shell (within $4 \mathrm{~mm}$ ) $+4 \%$ SQD-98 medium coarse + 3\% SQD-98 fine + 0.5\% JYW-2 + 1\% composite plugging agent III.

It can be seen from Table 1: When using the TL-1 high temperature and high pressure plugging instrument to evaluate the three plugging agents, when the displacement pressure is lower than $6 \mathrm{MPa}$, there is no leakage of plugging slurry; When the displacement force increased to $7 \mathrm{MPa}$, the leakage occurred, and the leakage was successfully plugged in $110 \mathrm{~s}$. Using traditional equipment to

Table 1. Comparison of plugging effects of different plugging instruments.

\begin{tabular}{rrrrrr}
\hline Instrument & $\begin{array}{r}\text { Crack Plugging } \\
\text { width/mm }\end{array}$ & $\begin{array}{r}\text { Leakage minimum } \\
\text { slurry }\end{array}$ & Lessure/MPa & Leakage time/s & $\begin{array}{r}\text { Cumulative } \\
\text { loss/ml }\end{array}$ \\
\hline TL-1 & 1 & $1^{*}$ & 7 & 125 & 600 \\
Traditional instrument & & & 4 & 160 & 1500 \\
TL-1 & 2 & $2^{*}$ & 7 & 110 & 560 \\
Traditional instrument & & & 5 & 150 & 1250 \\
TL-1 & 4 & $3^{*}$ & 7.0 & 90 & 900 \\
Traditional instrument & & & 3 & 120 & 2000 \\
\hline
\end{tabular}

Table 2. Comparison of the pressure-bearing capacity of the plugging slurry to form a plugging layer on different plugging instruments

\begin{tabular}{|c|c|c|c|c|c|c|c|c|c|}
\hline \multirow{2}{*}{ Instrument } & \multirow{2}{*}{$\begin{array}{l}\text { Plugging } \\
\text { slurry }\end{array}$} & \multirow{2}{*}{$\begin{array}{r}\text { Crack } \\
\text { width } / \mathrm{mm}\end{array}$} & \multicolumn{7}{|c|}{$\begin{array}{l}\text { Pressure changes in mud tanks } \\
\text { at different flowback pressures }\end{array}$} \\
\hline & & & 1 & 2 & 3 & 4 & 5 & 6 & 7 \\
\hline TL-1 & $1^{\# \#}$ & 1 & 0 & 0 & 0 & 0 & 0 & 0 & 7 \\
\hline Traditional instrument & & & 0 & 2 & 3 & 4 & 5 & 6 & 7 \\
\hline TL-1 & 2\#\# & 2 & 0 & 0 & 0 & 0 & 0 & 0 & 7 \\
\hline Traditional instrument & & & 0 & 0 & 3 & 4 & 5 & 6 & 7 \\
\hline TL-1 & $3^{\# \#}$ & 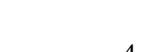 & 0 & 0 & 0 & 0 & 0 & 0 & 6.5 \\
\hline Traditional instrument & & 7 & 0 & 0 & 3 & 4 & 5 & 6 & 7 \\
\hline
\end{tabular}




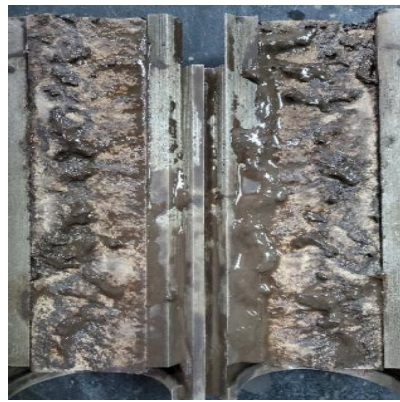

Figure 7 . The distribution state of $1^{\#}$ plugging slurry on the surface of core cracks.

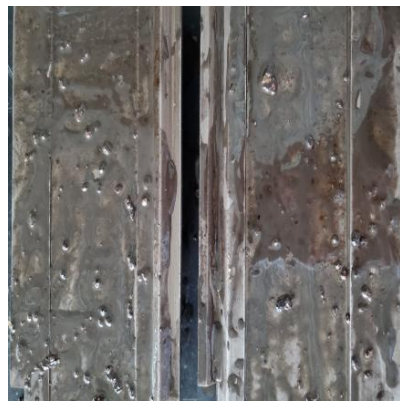

Figure 8. The distribution state of $1^{\#}$ plugging slurry on the surface of steel cracks.

evaluate $1^{\#}$ plugging $1 \mathrm{~mm}$ metal joints, the leakage starts to appear when the displacement pressure is $4 \mathrm{MPa}$, and the plugging is successful after $160 \mathrm{~s}$; When $2^{*}$ plugs $2 \mathrm{~mm}$ crack, the displacement pressure starts to appear when the displacement pressure is $4 \mathrm{MPa}$, and the plugging is successful after $160 \mathrm{~s}$; When $3^{\#}$ plugs $4 \mathrm{~mm}$ cracks, the displacement pressure starts to appear when the displacement pressure is $3 \mathrm{MPa}$, and the plugging is successful after $120 \mathrm{~s}$.

It can be seen from Table 2: When using the TL-1 high temperature and high pressure plugging instrument to evaluate the pressure bearing capacity of the three plugging layers, when the flowback pressure is lower than $6 \mathrm{MPa}$, the reading on the pressure gauge in the mud tank is zero; When the flowback pressure is increased to $7 \mathrm{MPa}$, the pressure in the mud tank slowly rises to $7 \mathrm{MPa}$. Using the traditional instrument to evaluate the pressure-bearing capacity of $1^{\#}$ plugging $1 \mathrm{~mm}$ wide metal cracks to form a plugging layer as $2 \mathrm{MPa}$, the pressure-bearing capacity of $2^{\#}$ plugging $2 \mathrm{~mm}$ cracks is $3 \mathrm{MPa}$, and the pressurebearing capacity of $3^{\#}$ plugging $4 \mathrm{~mm}$ cracks is $3 \mathrm{MPa}$.

It can be seen from Figure 7: Open the $1^{\#}$ plugging material to plug the core cracks of the TL- 1 high temperature and high pressure plugging instrument. It is found that the plugging material is more evenly distributed on the core surface and can form a dense and stable plugging layer in the cracks, therefore, the plugging effect and pressure capacity are better. It can be seen from Figure 8: Open the use of $2^{\#}$ plugging material to plug the stainless steel metal cracks of the traditional instrument, and it is found that only a small amount of large-size plugging materials are distributed on the surface of the metal cracks, small materials are basically leaked out under the action of pressure difference, and there is no plugging layer with different size material gradations formed on the leakage 
layer. Therefore, the plugging effect and the pressure bearing effect are not as good as the natural core fracture experiment. The main reason is that the metal surface is smooth, and the plugging material cannot be adsorbed or stuck, and the real effect of the formation cannot be simulated.

\section{Conclusion}

In view of the fact that the filter medium of the traditional plugging device cannot truly simulate the adsorption and hooking effect of the leaking stratum on the leak-proof plugging material, a high-temperature and high-pressure plugging device was designed. The filter medium of the instrument is a natural core with cracks of different sizes and a sand-filled bed of different sizes. The experiment temperature and pressure can be selected according to the formation conditions. The instrument can not only evaluate and select the preferred leak-proof and plugging materials, but also evaluate the preferred leak-proof and plugging drilling fluid system, study the pressure-bearing capacity of the plugging layer and the backflow pressure-bearing capacity of the plugging layer, and provide technical support for on-site construction.

\section{Acknowledgements}

First of all, I would like to express my deepest gratitude to my teacher, Professor Luo Chunzhi, who guided me in the design of the experiment and the writing of the thesis. Without her enlightening guidance and patience, this paper would not have reached its current form. I sincerely thank my classmates Zhang Chujun, Wang Baijing, Li Xueting, and Zhang Huan in the laboratory for their support and encouragement.

\section{Conflicts of Interest}

The authors declare no conflicts of interest regarding the publication of this paper.

\section{References}

[1] Yan, J.N. (2001) Drilling Fluid Technology. University of Petroleum Press, Dongying, Shandong, 146-148.

[2] Huang, H.J. (2009) The Development Status of Drilling Plugging at Home and Abroad. Engineering Technology and Industrial Economics, 4-5.

[3] Yu, H.F. (2014) Experimental Simulation of Plugging in Fractured Reservoirs and Optimization of Plugging Slurry Formulation. Master's Thesis, Southwest Petroleum University, Chengdu, 5-9.

[4] Kageson-Loe, N.M., Sanders, M.W., Growcock, F., et al. (2009) Particulate-Based LossPrevention Material-The Secrets of Fracture Sealing Revealed! SPE Drilling \& Completion, 24, 581-589. https://doi.org/10.2118/112595-PA

[5] Shi, B.Z., Hu, X.H., Gao, S.Y., et al. (2014) Visual Simulation Test and Evaluation of Micro-Fracture Plugging in Hard and Brittle Shale. Petroleum Drilling Technology, $42,32-33$. 